\title{
The Diagnosing of Vehicle Steering System by Video Recording Method
}

\author{
Fedor Smolenkov ${ }^{1 *}$, and Igor Blyankinshtein ${ }^{1}$ \\ ${ }^{1}$ Siberian Federal University, 79 Svobodny prosp., Krasnoyarsk 660041, Russia
}

\begin{abstract}
The possibility of vehicle steering system diagnosis based on analysis and visualization data processing is considered. The video recording method for the diagnostic troubleshooting of steering system and front suspension group is proposed. The method provides for placement of the motor vehicle on the diagnostic station, with further webcamera installation for measuring the steering wheel rotation angle and clear contract marking on the steering joint under experiment, setting the webcamera to fix and record the beginning of the movement steering joint under experiment, the video registration of the steering test results and further video processing using dedicated software. The outcomes of the evaluation of the method is delivered.
\end{abstract}

\section{Introduction}

When in operation a vehicle is exposed to shock stress, friction and other factors which essentially affect operability of all SS elements and the front suspension system FSS that lead to linkage side play. With the increase of linkage clearance in the SS and FSS elements, the sensible freedom of the wheels for displacement against the body of the vehicle which the operator may fail to control. Further wear out of movable linkages and incorrect adjustments of a steering gear assembly will lead to the increase of friction load in SS which, in turn, may account for a sudden destruction of steering linkage and complete loss of control over the vehicle and at the worst road accident (RA). According to RA statistics categorized by types of vehicle failure, SS and FSS failures account for $15 \%$ and $32 \%$ respectively $[1,2]$.

High quality preventive maintenance and proactive response to all kinds of vehicle malfunction will contribute considerably to decrease of RAs' rate. Therefore, the development of troubleshooting methods and algorithms in SS and FSS is seen as highly relevant and will be complemented by the active safety of a vehicle [3-7].

All failures and SS failures (figure 1) during vehicle operation are accompanied by noises, vibrations, knocks and general decrease in vehicle performance. Among the most common failures are linkage free plays of a FSS and SS. Taking into account physical nature of free play formation in movable FSS and SS and the nature of their manifestation in cinematic it is obvious that SS with their rotating motion, free play will manifest in angular units $\left(\Delta \varphi_{n}\right)$, in SS with inplane-parallel motion-metric units $\left(\Delta l_{m}\right)[8]$.

\footnotetext{
*Corresponding author: theo_espanol@mail.ru
} 
Continuous vehicle operation without adequate preventive maintenance and service results accounts for 15 to $20^{\circ}$ in SS total free play rate. Wears of SS various linkages results in increase of in a steering wheel free play by $2-20^{\circ}$ [1]. Therefore, the value of total SS free play is calculated based on all steering wheel free plays, caused by free plays in all elements and linkages which move in space in SS and FS:

$$
S=\left(\alpha_{1}+\beta_{1}+\ldots+\alpha_{n}+\beta_{n}\right)
$$

where $S$ - total steering wheel side plays $\mathrm{SS},{ }^{\circ}$;

$\alpha_{n}=f\left(\Delta \varphi_{n}\right)$-free steering wheel position, caused by a linkage free play in rotation $\mathrm{n},{ }^{\circ}$;

$\beta_{m}=f\left(\Delta l_{m}\right)$-free steering wheel position, caused by a linkage free play in plane-parallel motion, ${ }^{\circ}$;

$n$ - number of movable linkages in the elements of steering control in rotation;

$m$ - number movable linkages in the elements of steering control, which are in plant parallel motion.

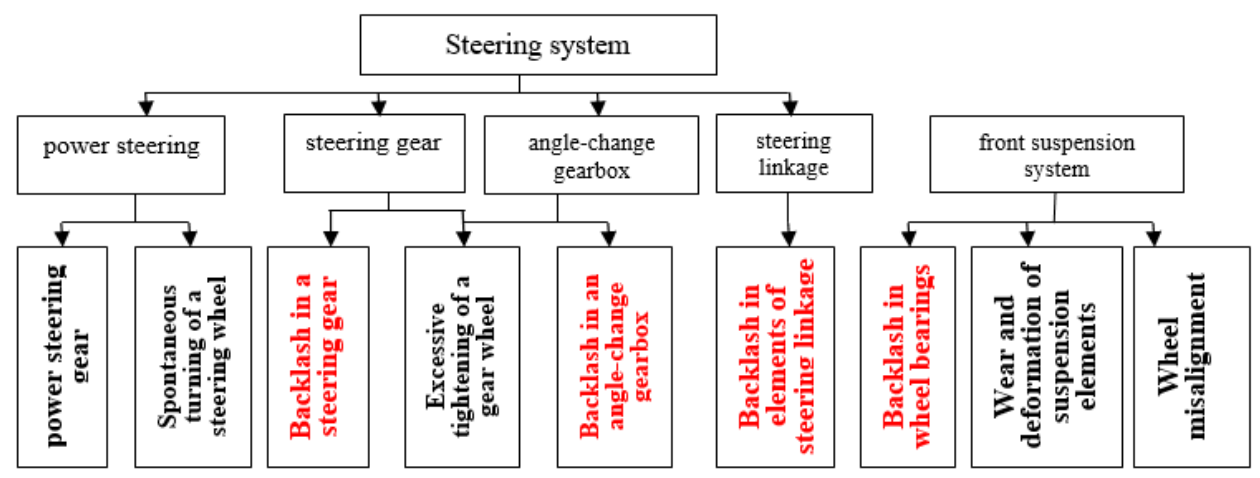

Fig. 1. Faults in SS and FSS.

In accordance with standard documents(SD) $[9,10]$ the basic diagnostics indicator is considered SS total free plays, which is steering wheel position at the startup of rotation controlled wheels in one direction to steering wheel position matching the startup of their rotation to the opposite direction which corresponds their straight-line motion of a vehicle.

The basic instrument to measure SS total free play rate is a tool - endfloatmeter.

Due to the high significance of the steering system and suspension system technical condition for the safety on road, the aim of the work is to create a technology of element-byelement diagnostics of these vehicle systems using video recording

\section{Material and methods: Existing methods and their disadvantages}

Let us consider the existing methods of diagnosing SS in terms of systematic approach (Figure 2) [11,12].

In order to measure the value of total free play in SS, diagnostics is made on the following order (Figure 2a):

- based on the requirement of $\mathrm{SD}$, an operator subject to controlled factor $P_{i}\left(x_{i}, y_{i}, z_{i}\right)$ of mutual positioning of a vehicle, measuring instruments and the operator, prepares and implement the test procedure, by following step-by-step instruction $S(t)$, which are displayed on a measurement unit.

- during the test, the operator performs control actions $U_{1}$, (rotation of the steering wheel); 
- the measuring tool (endfloatmeter) measures the steering-wheel angle $\mathrm{U}_{\mathrm{\pi}}$, and in the end, functional parameter $X_{1}$ (startup point for the steering wheel when it starts rotation);

- measurement results $R M_{1}$ (SS total side play) are displayed on the screen of the measurement unit.

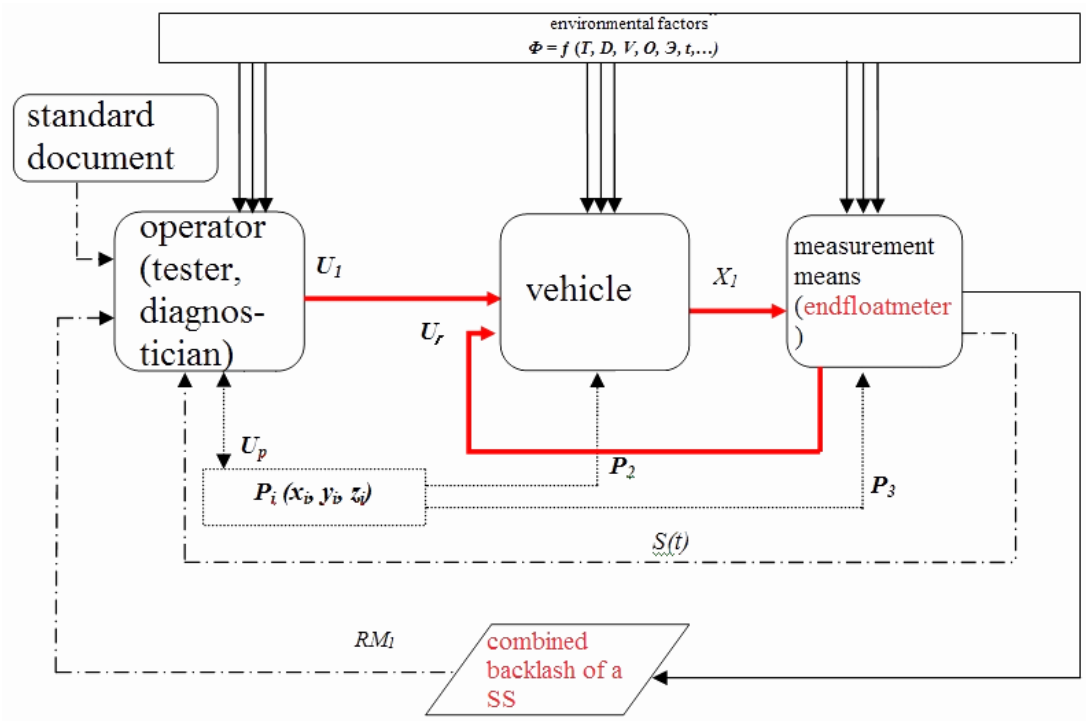

a

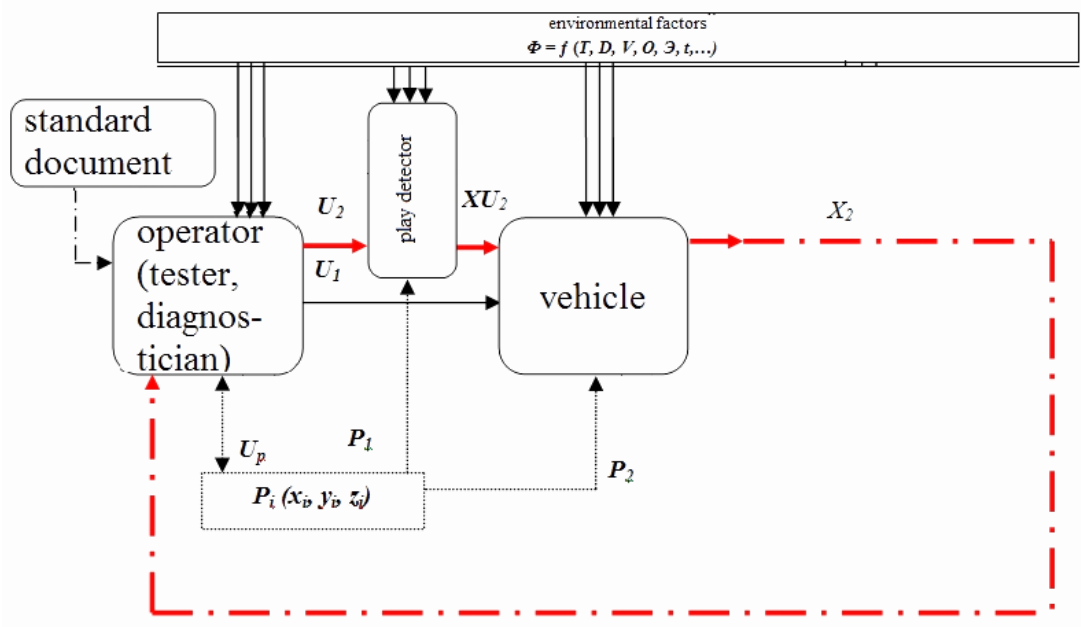

b

Fig. 2. Structural scheme for SS and FSS diagnostic: a) System 'Operator-Vehicle-

Endfloatmeter-Environment; b) System 'Operator-Play-detector-Vehicle_Environment'.

In order to measure the side play of some movable elements of a steering system, the diagnostics is implemented in the following way (Fig. 2b):

- the operator sends control actions to SS and FS elements (altering load)U1 or with the use of a controller of control actionsU2 (side play detector);

- the operator by the results of visual assessment (if the technical condition X1), and based on this organoleptic test makes a conclusion about the condition of the SS and FSS linkages. 
The analysis of the data of the structural schemes reviled the following weaknesses of currently applied methods for diagnostic of technical condition of a SS and FSS of a vehicle:

- low reliability of the test results, which rests on a subjective nature of visual measurement of operability,

- inability to precisely measure the value of side play for each tested linkage in metric units,

- inability of on-line data saving.

\section{Theory}

\subsection{Proposed method}

IT technologies may contribute to solving the problem by means of hardware and software complex (further -'Complex') designed to test a SS and FSS, structural scheme of which is shown in Fig. 3. This Complex was developed in the Department of Transport of the Polytechnic Institute of the Siberian Federal University [8,13-15]. The computer-based diagnostic technology provides for marking adjacent elements of movable linkages of a SS and FS with contrast paint, video recording of forced motion of SS and FS elements in space. Then, based on the results of the video recording, which is processed with the developed software provides a conclusion on the technical condition of the linkages.

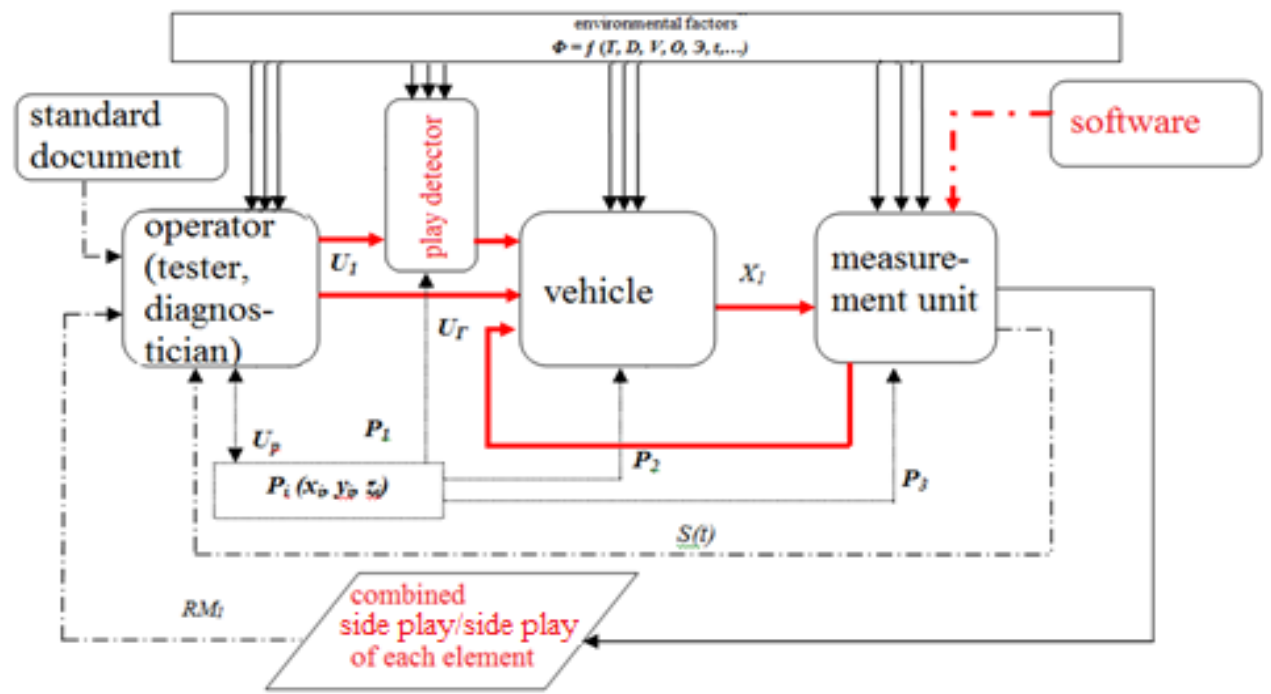

Fig. 3. Structural scheme of the system «Operator- Play-detector-Vehicle-Environment».

When alternating loads are applied to SS and FS elements, the video recording is arranged in a way to have both contrast marks within the frame. 


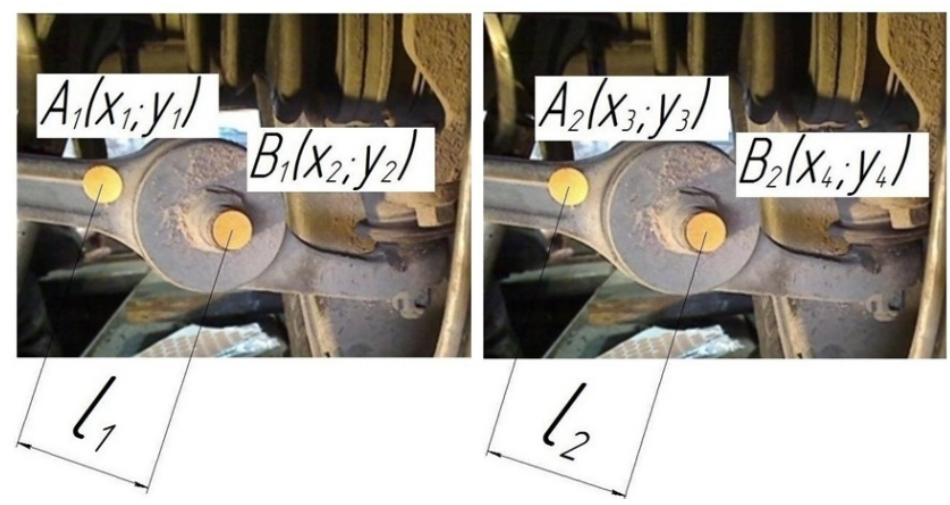

Fig. 4. Frames of a video, on which joints are in their end positions.

Then, computer-generated imagery is analyzed with the use of elements of pattern recognition (Fig.4) the values of the distances between marks $(11,12)$ are considered according to their coordinates in the end positions of the joints:

$$
l_{1}=\sqrt{\left(x_{2}-x_{1}\right)^{2}+\left(y_{2}-y_{1}\right)^{2}} ; l_{2}=\sqrt{\left(x_{4}-x_{3}\right)^{2}+\left(y_{4}-y_{3}\right)^{2}} .
$$

The side play amount in the linkages $\Delta l$ is identified on the basis end positions of the linkages by calculation of maximal differences of marks' positions:

$$
\Delta l=\max \left[l_{1}-l_{2} ; l_{2}-l_{1}\right] .
$$

Then, the calculated amount is compared against the standard amount. Therefore, the concussion about a technical condition of controlled linkages of FSS and SS elements is as follows:

$$
\Delta l \leq \Delta l_{H}-\text { the condition is } \mathrm{OK} ; \Delta l \geq \Delta l_{H}-\text { the condition is not } \mathrm{OK} \text {; }
$$

where $\Delta l_{H}-$ is a calculated maximum standard limit for the side play.

\subsection{Approbation}

This method may be adapted for diagnostic of a vehicle's SS and FS by elements. In this case the procedure is arranged as follows (e.g. bus MAZ-103). The vehicle is placed on a test station so as to provide free access to all elements of steering management elements.

Then with the use of a universal holder the first webcam is placed on the steering wheel (SW). A screen with two contrast marks on it is placed in front of the webcam in a way that both marks are visible in the frame of the webcam (Figure 5a, b). Contrast marks are made on SS and FS linkages under test for the side play (Figure 6a). Each SS and FSS linkage is diagnosed separately. The amount of a side play in each linkage is measured in accordance with the amount of steering-wheel angle. In series in front of each linkage with the use of a magnetic corbel on a frame, the second webcam is placed in a way to that the marks are within the frame of the webcam (Figure 6b). The webcams are connected to a laptop, the software (Figure 7), allows to simultaneously processes videos from both webcams. The steering wheel angle is measured by the changes of marks on the video from the webcam placed on the SW. The beginning of a linkage motion is measured by the changes of a mark on the video from the webcam placed on the linkage.

Thus, in order to measure the side play of a linkage, the SW shall be rotated, firstly, in one direction until a tested linkage starts moving (for example, rotation of a drive shaft 
(Figure 6)), then in the opposite direction. The amount of steering-wheel angle will be the value of the side play of the first linkages tested. The software measures the value of a side play in the linkage, which is expressed in degrees of steering-wheel angle for each movable linkage. Taking into account design specifications of a SS of a vehicle, as well as a nature of side play formation in each linkage, a mathematic model of accumulation of a free angular motion of steering wheel in a function of movements of SS movement elements (1) is built. This model allows to measure amount of linkage in each linkage in metric units. The results are displays on the screen of a laptop.

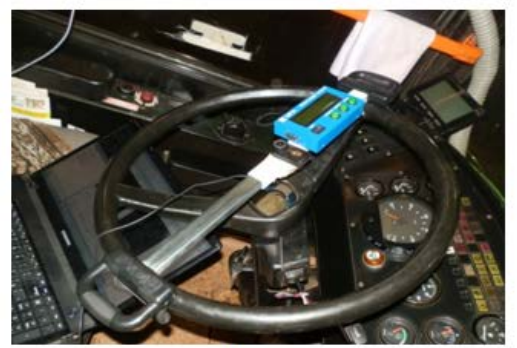

a

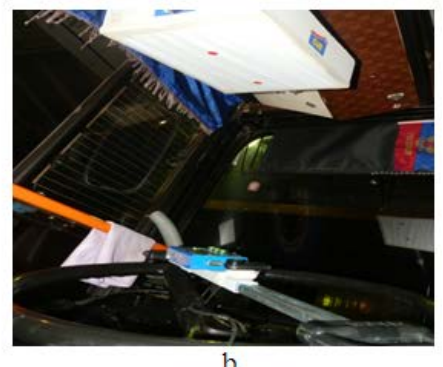

b

Fig. 5. Placement of equipment on a steering wheel: a) Placement of webcam on a steering wheel; $b$ ) Placement of a marked screen.

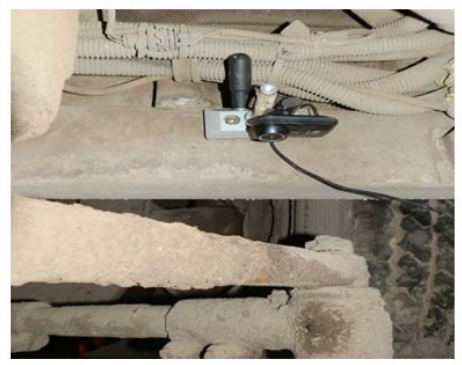

a

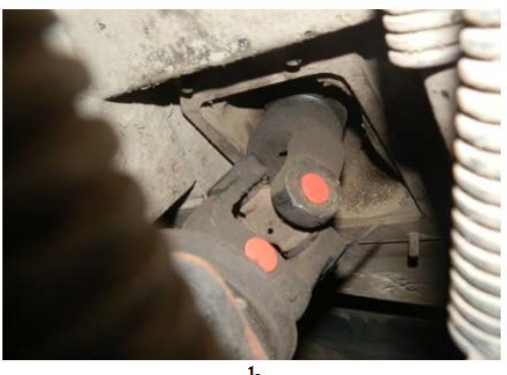

b

Fig. 6. Placement of equipment and tools on the linkages intended for testing: a) Placement of a webcam on a tested linkages; b) A tested linkage with marks.

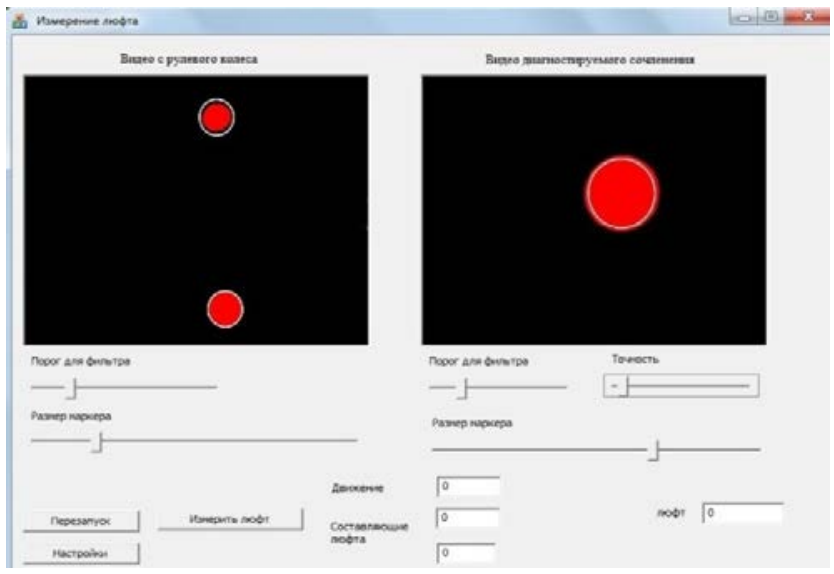

Fig. 7. Interface of software that analyses videos from a webcam. 
In order to check the sensitivity of the proposed method (lower measurement level) we identified the precision for side play measurement by a webcam. For this a scheme for determination of the start of mark movement in focus of a webcam (Figure 8). Webcam 1 is mounted on a tripod, a screen with a mark 2 was placed in front of it on indicator 3 , to have the mark clearly visible in the frame in order to put the mark right within the frame of the webcam. The measurement process was started by startup of the software and slow rotation of drive screw 4 of the indicator. A hung screen was moving downwards up to the moment of the image shift actuation of the software and acoustic signal.

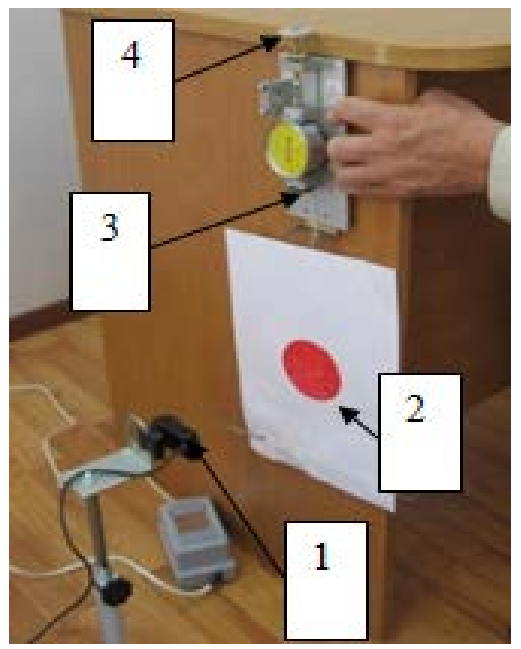

1 - webcam, 2 - screen with a mark, 3 - indicator, 4 - drive screw of the indicator

Fig. 8. Measurement accuracy test.

\section{Results and Discussion}

The results of approbation developed technology for monitoring parameters of the SS are presented below in Table 1 and Figure 9. The recorded values of linear marked screen movement were registered in a table 1.

Table1. Results of sensitivity measurement.

\begin{tabular}{|c|c|c|}
\hline $\begin{array}{l}\text { Webcam-to-screen } \\
\text { distance, } \mathrm{mm}\end{array}$ & $\begin{array}{c}\text { Red circle dia., } \\
\text { mm }\end{array}$ & $\begin{array}{l}\text { Sensitivity of the motion start-up } \\
\text { according to the dial test indicator, } \mathrm{mm}\end{array}$ \\
\hline \multirow[t]{10}{*}{ 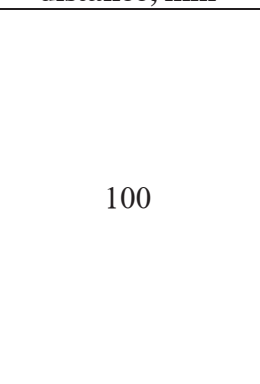 } & \multirow[t]{10}{*}{ 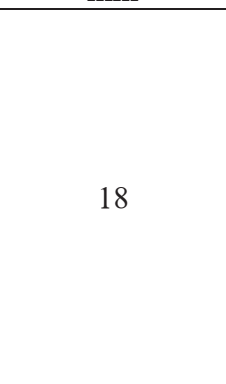 } & 0.09 \\
\hline & & 0.13 \\
\hline & & 0.14 \\
\hline & & 0.12 \\
\hline & & 0.15 \\
\hline & & 0.13 \\
\hline & & 0.09 \\
\hline & & 0.16 \\
\hline & & 0.15 \\
\hline & & 0.10 \\
\hline \multicolumn{2}{|c|}{ Averagevalue } & 0.126 \\
\hline \multicolumn{2}{|c|}{ Parameter spread $\Delta=$ max-min } & $0.16-0.09=0.07$ \\
\hline \multicolumn{2}{|c|}{ Root-mean-squaredeviation } & 0.0254 \\
\hline \multicolumn{2}{|c|}{ Dispersion } & 0.000649 \\
\hline
\end{tabular}


This data show, that recorded sensitivity of marks movement controlled by a webcam suggests an opportunity of practical use of the developed technology for diagnosing of SS and FSS linkages of a vehicle by the means of video recording.

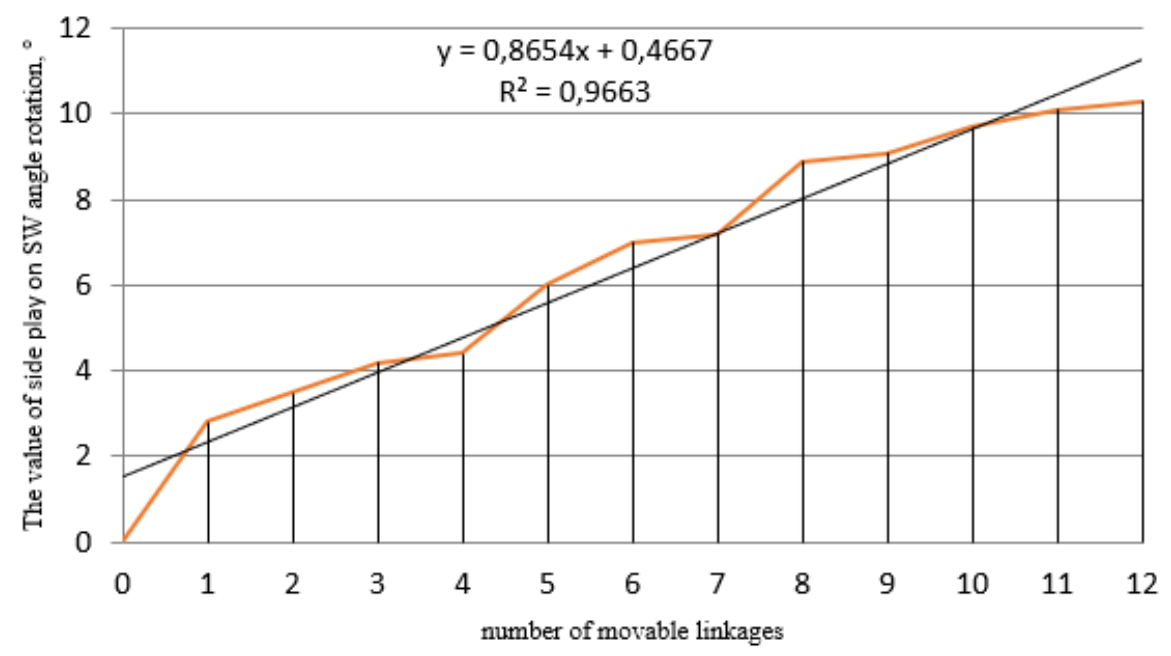

Fig. 9. The nature of accumulation of total side play on SW depending on joints.

The total side play of SS, measured by the proposed method, amounted to $10,3^{\circ}$ (Fig.9). Which is consistent with the results of measurements total side play by a certified enfloatmeter META ISL-M.

This method will make it possible to determine the nature of side play in SS. This will reduce the number of accidents on roads and increase the active safety of a vehicle.

\section{Conclusions}

In present work, we presented the technology of determining the technical condition of the elements of SS and FSS based on video recording. As the advantages of this diagnostic method, based on processing of visual information can be credited a possibility to measure the amount of total and individual side play of SS and FSS in metric units $\left(\mathrm{mm},{ }^{\circ}\right)$; elimination of subjectivity in assessment of technical state of controlled linkages; efficiency of diagnostics in respect of time saving and high precision of diagnosing; possibility of online recording and storage of diagnostic results.

\section{References}

1. N. Ya. Govorushchenko, Technical operation of vehicles. Kharkov: Higher School. Kharkov University Public House; (1984).

2. A.V. Myrochkin, Development of system for operability assurance of front suspension steering management systems for vehicles. Candidate's Thesis in Engineering; Vladimir, (2010).

3. P. Metallidis, G. Verros, S. Natsiavas, Papadimitriou C. Fault detection and optimal sensor location in vehicle suspensions. J. Vibration Control; vol. 9, no. 34, pp. 337359. (2003) 
4. A. Chamseddine, Y. Noura, Control and Sensor Fault Tolerance of Vehicle Active Suspension. IEEE Trans. Control Systems Technology; vol. 16, no. 3, pp. 416-433. (2008)

5. S.D. Arogeti, C.B. Wang, M.Yu. Low, Fault detection isolation and estimation in a vehicle steering system. IEEE Trans. Ind. Electron; vol. 59, no. 12, pp. 4810-4820. (2012)

6. Yu. Ming, W. Danwei, Model-Based Health Monitoring for a Vehicle Steering System with Multiple Faults of Unknown Types. IEEE Trans. Ind. Electron; vol. 61, no. 7, pp. 3574-3586. (2013)

7. V.V. Lyandenburgski, On-board diagnostic system for steering management system of vehicles. Internet newsletter SCIENCE STUDIES; vol. 9, №2. (2017)

8. F.Yu. Smolenkov, I.M. Blyankinshtein, A.S. Kashura, A.M. Askhabov Estimation of constituent of total side play for the steering management for bus MAZ -103. Transport systems of Siberia. Development of the transport systems as a driver of the national economy growth. Krasnoyarsk: International Research and Practical Conference; Part 2, pp. 552-559. (2016)

9. TR CU 018/2011Technical regulations of the Customs Union on wheels transport vehicles safety, approved by the Decision of the CU Commission as of December 9, № 877. (2011)

10. GOST R 51709-2001 Transport Vehicles. Safety and technical condition Requirements and assessment methods. Moscow: Standardinform, (2008)

11. I.M. Blyankinshtein, Scientific base for improvement of process and maintenance equipment of vehicles. Doctoral Thesis in Engineering; Irkutsk, (2013)

12. R. Isermann, Model-based fault-detection and diagnosis - status and applications. Annual Reviews in Control, vol. 29, no. 1, pp. 71-85. (2005)

13. I.M. Blyankinshtein, F.Yu. Smolenkov, Technology of diagnosis linkages of suspension elements. Special features of operation and maintenance of vehicles under hush climate conditions of Siberia and Far North. Problems with certification, diagnostic and technical condition management. Irkutsk: Eighty third International Scientific and engineering Conference of the Automobile Engineering Association; pp. 157-163. (2013)

14. I.M. Blyankinshtein, F.Yu. Smolenkov Examination of the technical state of steering management of the element for busses MAZ-103. An Automobile for Siberia and Far North. Design, operation and maintenance and economic efficiency. Irkutsk: Ninetieth International Scientific and engineering Conference of the Automobile Engineering Association; pp. 334-340. (2015)

15. I.M. Blyankinshtein, F.Yu. Smolenkov Method for diagnostic technical condition of front suspension elements in vehicles. Patent № 2537211; The Russian Federation, IPC G01M17/04, (2014) 